\title{
Acting Egoistically in a Crisis: How Emotions Shape Data Donations
}

\author{
Luisa Pumplun \\ Technical University of Darmstadt \\ luisa.pumplun@tu-darmstadt.de
}

\author{
Amina Wagner \\ Technical University of Darmstadt \\ amina.wagner@tu-darmstadt.de
}

\author{
Christian Olt \\ Technical University of Darmstadt \\ christian.olt@tu-darmstadt.de
}

Anne Zöll

Technical University of Darmstadt Anne.zoell@tu-darmstadt.de

\author{
Technical University of Darmstadt \\ peter.buxmann@tu-darmstadt.de
}

Peter Buxmann

\begin{abstract}
The spread of COVID-19 has affected all of us, be it financially, socially, or even physically. It has caused uncertainty and anxiety, which has put people into a "hot" mental state. Referred to as an empathy gap, people are assumed to make emotion-driven decisions in "hot" states compared to "cold" states, which contrasts with the normative assumption of rational decision-making in privacy research. Based on an experimental survey study among 445 participants, we investigate whether people's mental state interacts with individuals' information disclosure decision-making. We measure our research model in the context of actual health data donation, which constitutes a critical surveillance factor in the COVID-19 crisis. Thereby, we contribute to research by (1) investigating data donation behavior amid a crisis and (2) helping to explain further nuances of privacy decision-making and the importance of trust as a context-dependent driver of data donation.
\end{abstract}

\section{Introduction}

In the years 2020 and 2021, the coronavirus infected millions of people worldwide. At the time of writing this paper, more than 3.7 million died because of it. To combat the rapid spread of COVID-19, governments worldwide implemented countermeasures like closing educational institutions and restricting public life. Some even imposed a lockdown and forced their citizens to stay at home for several weeks [4]. Information systems have played a significant role in managing various crises [e.g., 27]. Research institutes and governments could particularly benefit from information systems that gather and store people's health data to predict and control the global spread of COVID-19 today-but also in the following years [61]. Accessing personal health data in real-time and processing it with the help of data analytics techniques (e.g., machine learning) promise new opportunities to measure and predict the spread of COVID-19. However, for such dataanalytical endeavors to be successful, research institutes rely on the population's health data [4]. Thus, to contribute to the current COVID-19 pandemic, this manuscript revolves around individuals' data donation decisions. Data donation describes the act of people willingly disclosing their personal health information for research institutes with no apparent flow of value in return $[66,76]$. During the COVID-19 pandemic, many institutions attempt to collect and store personal health information. For instance, research institutes offer smartphone applications, which enable users to voluntarily share their current health status by answering questions, for example regarding their body temperature [e.g., 24]. Other applications rely on fitness data collected by fitness-trackers to detect infectious diseases even before users show first symptoms. Even though these emerging technologies seem to be promising, they spread slowly among the population, which counteracts their effectiveness [34]. Therefore, it is of utmost importance to understand individuals' decision-making to donate health data to better support the public health system. However, research currently does not provide guidance on how people form data donation decisions, especially during a crisis such as the COVID-19 pandemic. Previous research mainly relies on the privacy calculus [21, 40] to explain the antecedents of information disclosure [e.g., 6, 39]. In this vein, established privacy research characterizes internet users as rational agents capable of making rational disclosure decisions [21, 69]. However, a small body of research based on behavioral economics started to question individuals' capability of making rational decisions regarding their privacy [e.g., 3, 5, 13, 22]. They provide evidence that behavioral biases like immediate gratification [1], overconfidence [72], and stereotypical thinking [31] 
lead to corresponding errors in privacy-related decisions.

Pandemics cause heightened uncertainty levels among the affected population leading to stress, anxiety, and frustration as an emotional response [10, 53]. Faced with the COVID-19 pandemic, we expect an empathy gap to distort individuals' rational and stable disclosure decisions. Empathy gaps occur if people fail in behaving in accordance with their cold mental state (low intensity of emotions) when being in a hot state (high intensity of emotions) [12, 43, 48]. Specifically, if the intensity of emotional influences arises, these emotions drive intuitive, automatic, and spontaneous actions rather than rational ones $[42,43$, 67]. Similar to how the word hate triggers negative emotions [20,67], asking people to donate data for research on a current pandemic can put people in a hot state and, in turn, distort rational decision-making. In contrast, people who make a donation decision for a more distant (less emotionally arousing) research purpose are in a cold state and act more calmly, rationally, and receptive $[43,68]$ as formerly proposed by privacy research [21]. Motivated by investigating further nuances of the privacy calculus [22] and guided by behavioral economics research [42, 43, 68], our research questions (RQ) are:

RQ1: Are data donation decisions formed differently when people are in a cold state compared to a hot state?

RQ2: And if so, how do these states influence the magnitude of actual data donation behavior?

To answer both research questions, we conduct an experimental survey study among 445 participants. We manipulate respondents' mental state by asking participants to donate data for either research on the current threat of COVID-19 (causing a hot state) or Ebola as a more distant research purpose (causing a cold state). Specifically, we test the effect of the mental state on the link between institutional trust and data donation behavior and the relationship between privacy risks and data donation, respectively. We show that trust is only significantly related to data donation behavior for research on Ebola (cold state). In the case of COVID-19 (hot state), participants focus primarily on privacy risks that inhibit self-disclosure. Therefore, the magnitude of actual data donation in a hot state is lower than in a cold state.

Understanding how mental states influence data donation decisions is a crucial and timely societal topic that provides several implications. We highlight that - amid a crisis - research institutes that rely on real-time health data should promote a strong image of privacy-friendliness to lower perceived privacy risks. Surprisingly, trustworthiness does not positively affect people's decision to donate data while in a hot state, lowering the total amount of data donated to crisisrelated causes compared to non-crisis-related donation purposes. In the long run, research institutes should establish a continuous, more passive data gathering process that allows collecting data across crises and non-crises periods. Moreover, even though fear appeals effectively motivate people to follow governmental rules [63], it is also necessary to mark COVID-19 not only with negative emotions causing a hot state but also with hope and social cohesion to strengthen prosocial behavior. Furthermore, we contribute to privacy research by exploring contextspecific disclosure decisions. Hence, our findings contribute to the literature on paradoxical information disclosure decisions and respond to the need to investigate data donation decisions amid a crisis. We particularly provide insights on reasons for contradictory study results regarding the linear link between trust and disclosure decisions $[35,55]$.

\section{Theoretical Background}

Research on information privacy typically assumes that Internet users disclose personal information based on a rational trade-off between their benefits, privacy risks, and institutional trust [16, 21, 39]. Referred to as the privacy calculus, Internet users release their personal information only if benefits exceed perceived privacy risks [21, 77]. Simultaneously, users' institutional trust may countervail privacy risks and thus leverage information disclosure [21]. While ones' benefits are immediate and vary across different contexts [69], users' perceptions of privacy risks and trust are the major overarching influential factors for information self-disclosure [21, 73, 77].

Data donation constitutes a sub-form of selfdisclosure where individuals consciously decide to voluntarily disclose their personal health related information to a collective dataset for free to support research institutes $[11,66]$. Driven by altruistic motives, similarities can be drawn between donating data, blood, or organs for the benefit of others' health [66]. Especially in the context of data donation, privacy risks and trust perceptions should dominate data donation decisions since neither an exchange of value occurs nor are benefits offered in return for releasing personal information. Instead, the benefits of donation are more complex, distant, and abstract in nature (e.g., supporting research) [64]. To investigate trust and privacy risks perceptions in more ambiguous situations [55], we analyze the extent to which an empathy gap leads to differing trust and privacy risks assessments. 
Cold and hot states delineate the duality between the cold rational and the hot emotional parts of ourselves. It results from individuals' inability to empathize with their different states, which is termed an empathy gap $[12,43]$. Making decisions in cold and hot states leads to different behavioral outcomes [42, 43]. People deliberately analyze all available information in a cold state to arrive at a rational decision [42, 43, 48]. People's decision-making is guided by reasons, logical connections, and past experiences [48, 67]. They make conscious decisions based on their long-term preferences [48]. This perspective is also applied in privacy research, where people are assumed to make a rational analysis to arrive at a disclosure decision [16, 21]. However, psychologists emphasize that as soon as the intensity of the felt emotion arises, it reduces individuals' motivation to process all available information rationally $[42,43]$. Instead, individuals act based on their gut feelings and following their acute short-term goals [48, 67]. Having intense emotions puts users in hot states where information is processed automatically, intuitively, and driven by emotional factors, which lead to a spontaneous decision [43]. These intense emotions occur if a stimulus triggers emotions associated with that specific object [67].

Indeed, donation research has shown that emotional stimuli can influence donation behavior. For instance, within the organ donation context, Kopfman \& Smith (1996) [38] provide evidence that non-holders of organ donor cards are primarily fearful of negative consequences attached to the sign-up for organ donation. Even though their study participants state a high intention to sign up for a donation card and have a positive attitude towards organ donation, they finally fail to transform their intention into action. Several scholars discuss in this context how external stimuli of "sensationalistic, negative media portrayals" [51:674] amplify fears related to organ donation. For instance, spreading myths about prematurely declared deaths or corruption in the allocation of organs eventually leads to fewer organ donations [51]. Due to the current shortage of organ donors, researchers analyze how to raise awareness and reduce negative emotions related to organ donation using social media [52]. A similar phenomenon is shown in the context of blood donation, where potential donors reject blood withdrawal due to their fears related to the process [29]. Specifically, confronting potential donors with the critical decision to donate blood can evoke anticipatory anxiety associated with the "fear of blood, needles, pain, and fainting" [29:114]. Therefore, powerful negative emotions can hinder people from making sound decisions based on their long-term preferences (e.g., helping others) [42].
Even though donating organs or blood differs from the context of data donation, these findings provide first insights into possible phenomena related to emotions and donation behavior. Indeed, "one of the central emotional responses during a pandemic is fear" [10:461], potentially mitigating the donation behavior for research on COVID-19. Building on the above assumption that a negative emotional stimulus interacts with donation decisions, we extend the privacy calculus by investigating how different mental states influence data donation decisions.

\section{Hypotheses Development}

Defined as users' worry about losing control over their personal information [46], privacy risks have been studied as the major impediment associated with information disclosure [6, 39, 77]. However, prior privacy studies on the link between privacy risks and disclosure are inconsistent [13, 22, 35]. Recently, this influence is shown to be affected by emotions present at the time of decision-making [35, 41]. For instance, Alashoor et al. (2018) [5] investigated the effect of an individual's mood state on the link between perceived privacy risks and the intention to disclose information. They found evidence that negatively inclined users take greater account of perceived privacy risks. Indeed, people are more likely to narrow their attention on risks if an emotional stimulus triggers negative feelings [42]. In this regard, emotions constitute an essential means of information [32, 58]. For instance, being anxious signals that something might be wrong and promotes attention to potential risks $[5,67]$. With regard to the COVID-19 pandemic, people have intense negative feelings such as financial uncertainty, frustration, depression due to social isolation, or even fear of one's death $[10,14,53]$. Therefore-similar to how the word war triggers intense negative emotions [20, 68]-the research purpose COVID-19 might serve as an emotional stimulus that evokes strong negative emotions and consequently puts people in a hot state. These intense negative emotions might hinder individuals' willingness-to-donate their data. In this vein, individuals narrow their attention, act more egotistically based on self-related fears, and cannot account for others' needs [12, 42, 43]. As a result, if one is asked to donate health data to institutes researching COVID-19, we presume that people have a higher disposition to privacy risks and are less willing to donate data. In contrast, concerning less emotional research purposes, people are less likely to be biased towards risks as they act in a relatively calm situation (a cold state) where risks seem less salient [58]. Therefore, we postulate that being in a hot state 
leverages the effect of privacy risks on data donation behavior:

H1: The direct link between privacy risks and data donation behavior will be stronger in hot states.

Defined as an individual's belief that the other party is competent, benevolent, and honest, trust has been regarded as a decisive determinant to disclose personal information $[21,47]$. While a great body of studies could substantiate the trust-disclosure link [8, $9,49,73]$, others indicate that trust is not significantly linked to disclosure decisions $[36,55]$. The reasoning behind this is that the role of trust depends largely on the concrete institution in which trust is placed, on the time, place, and circumstances underlying the decision, and on the emotions attributed to a particular situation $[37,50,73]$. In this context, intense negative emotions such as anxiety can impair the relevance of trust $[9,19]$. For example, put yourself in the scenario that you would like to do a bungee jump for the first time. You select a renowned provider with whom thousands have already taken the leap. In advance, you would rate the provider to be trustworthy. However, when you find yourself standing on the cliff, this previously granted trust might be irrelevant. Instead, your fear of heights prevents you from considering trust for your decision. Therefore, the effect of trust that has been granted based on rational expectations and available evidence in a calm situation [37] cannot simply be transferred to a more emotionally charged setting [50]. Similarly, facing the COVID-19 pandemic, individuals are confronted with inevitable financial uncertainty, infection fears, and social isolation, which represent a completely new, so far unknown extreme situation with intense negative emotions attached $[10,53]$. In such a scenario, the actors cannot draw on existing knowledge to extrapolate paths for rational decisions as the future is insecure $[43,50]$. Since there are no proven routines to rely on and fear determines actions, individuals no longer rely on trust but emphasize other, more intuitive, and salient antecedents to decide on their behavior [50]. Therefore, while in a decision-making situation that is less emotionally charged and characterized by little uncertainty (cold state), one's behavior strongly depends on the established trust, we assume that this determinant has less influence on the decision to donate data in a hot state:

H2: The direct link between institutional trust and data donation behavior will be weaker in hot states.

\section{Methodology}

To test our hypotheses empirically, we designed an online survey, which is a widely used methodology within privacy research [e.g., 23]. At the beginning of the survey, we explained the study's purpose to every participant and promised full anonymity of all respondents' answers. The survey consists of two parts: First, we asked all participants to donate health data to us as a research institute (i.e., a university) to combat the spreading of a virus. In this vein, we were able to measure actual data donation behavior. Second, we ran a questionnaire consisting of our main measurements, demographics, and controls. We debriefed all participants at the end.

Based on the preceding arguments, we presume that the research purpose triggers emotions putting participants in different mental states depending on the emotions' intensity. Hence, by varying the purpose of the data donation as an emotional stimulus, we deem to manipulate the intensity of emotions and, thus, the mental state of participants that shapes the decision to donate data. For every participant, we randomly assigned either fighting against COVID-19 or Ebola as the data donation purpose and included a short informative description of the respective virus (i.e., the current level of dissemination, the existence of vaccine). We chose Ebola as the second research purpose for two reasons: First, the disease is comparable to COVID-19, both medically and in terms of countermeasures. Notably, countermeasures like data collection applications have been first used during the Ebola epidemic [63]. Second, Ebola is expected to evoke less intense emotions in European participants because it has been particularly prevalent in West Africa from 2013 to 2016, has a lower risk of transmission, and an effective vaccination exists already [53]. In contrast, we assume that COVID-19 causes a hot state among participants as it is highly transmissible, infects people worldwide, countermeasures are still active, and no licensed vaccination had been developed during the time of our study. To test the success of the manipulation, we included a manipulation check on the intensity of emotions attached to the data donation purpose. We leaned on the negative affect scale from the PANASX list from Watson \& Clark (1999) [74] to distinguish the emotional response triggered by each scenario. After having read the welcome page along with the research purpose, we asked respondents to rate the following four statements along a 7-point Likert scale: (1) COVID-19/Ebola distresses me, (2) I am afraid about the spreading of COVID-19/Ebola, (3) Thinking of COVID-19/Ebola makes me nervous, and (4) COVID-19/Ebola upsets me. Being solely asked about their intentions to behave, participants frequently fail to predict their actual behavior. This intentionbehavior gap is particularly pervasive in privacy research [54]. To overcome this limitation of the privacy domain, we measure actual data donation 
behavior. Inspired by Acquisti \& Grossklags (2005) [2], we asked 14 different health-related questions of varying sensitivity, adapted from existing health data donation apps [e.g., 62]. Our list included questions on basic health data (e.g., body weight), lifestyle data (e.g., drinking behavior), medical history (e.g., medication), as well as on virus-specific data (e.g., frequency of coughing). For every question, the participants could either answer to make a donation or refuse to respond and thus decline to donate. Hence, we measure how many pieces of health information every participant chose to donate. For ethical reasons, we did not store the individuals' health data donated to us at any time but only counted how many questions were answered (a number between 0 and 14). After measuring actual behavior as a dependent variable, participants answered a questionnaire with different measurement scales to assess their actual data donation determinants. All measurement scales are based on prior literature and are adapted to fit the context of data donation. We list our main scales in Table 1. The participants' answers are scored along a 7-point Likert scale ranging from strongly disagree to strongly agree. Furthermore, we included the following control variables into our questionnaire, which are based on established measurement instruments: age, empathy as a trait [17], personal feeling of informedness about the virus [70], prior privacy experience [77], as well as general perceived health [6]. We included several preventives to counteract possible common method bias (CMB) stemming from the survey-based methodology [57, 59]. Moreover, a theoretically unrelated variable, namely fantasizing, was included as a marker to allow for testing for CMB retrospectively [57]. To ensure that we only included attentive participants in the analysis, we integrated two attention checks.

Table 1. Measurement Scales

\begin{tabular}{|c|c|c|}
\hline Var. & ID & Item \\
\hline \multirow{4}{*}{ 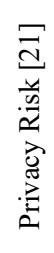 } & PR1 & $\begin{array}{l}\text { I am concerned that my donated health records } \\
\text { could be misused. }\end{array}$ \\
\hline & PR2 & $\begin{array}{l}\text { I am afraid that others might gain access to my } \\
\text { donated health data. }\end{array}$ \\
\hline & PR3 & $\begin{array}{l}\text { I am concerned about donating my health data, } \\
\text { because of what others might do with it. }\end{array}$ \\
\hline & PR4 & $\begin{array}{l}\text { I am concerned about donating my health data, } \\
\text { because it could be used in a way I did not foresee. }\end{array}$ \\
\hline \multirow{5}{*}{ 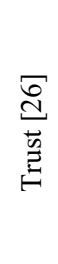 } & TS1 & This university is trustworthy. \\
\hline & TS2 & $\begin{array}{l}\text { This university wants to be known as one who } \\
\text { keeps promises and commitments. }\end{array}$ \\
\hline & TS3 & $\begin{array}{l}\text { I trust this university keeps my best interests in } \\
\text { mind. }\end{array}$ \\
\hline & TS4 & $\begin{array}{l}\text { This university has more to lose than to gain by not } \\
\text { delivering on its promises. }\end{array}$ \\
\hline & TS & This university's behavior meets my expectations. \\
\hline
\end{tabular}

We collected data from May to June 2020 in Germany with the assistance of a market research institute. Involving a market research institute enabled us to recruit participants who were not previously primed with the survey topic or originated from our network, potentially leading to biased results [44]. We included quotas (per manipulation group) to receive a data sample representing the average European Internet user [25]. Before analyzing the data, we dropped 17 cases because those respondents failed the attention checks. The resulting sample included 445 participants; 227 cases on COVID-19 and 218 cases on Ebola research. The characteristics of the sample can be found in Table 2 .

Table 2. Characteristics of Sample

\begin{tabular}{|c|c|c|c|}
\hline \multicolumn{2}{|c|}{ Characteristic } & \multirow{2}{*}{\begin{tabular}{|c|}
$\begin{array}{l}\text { COVID-19 } \\
(\mathbf{n}=\mathbf{2 2 7})\end{array}$ \\
$25.6 \%$ \\
\end{tabular}} & \multirow{2}{*}{\begin{tabular}{|l}
$\begin{array}{l}\text { Ebola } \\
(\mathbf{n}=\mathbf{2 1 8})\end{array}$ \\
$34.9 \%$
\end{tabular}} \\
\hline \multirow{5}{*}{ Age } & $18-30$ & & \\
\hline & $31-40$ & $29.9 \%$ & $22.4 \%$ \\
\hline & $41-50$ & $21.6 \%$ & $22.1 \%$ \\
\hline & $51-60$ & $11.9 \%$ & $14.6 \%$ \\
\hline & $>60$ & $11.0 \%$ & $6.0 \%$ \\
\hline \multirow{2}{*}{ Gender } & Male & $55.5 \%$ & $53.7 \%$ \\
\hline & Female & $44.5 \%$ & $46.3 \%$ \\
\hline \multirow{5}{*}{$\begin{array}{l}\text { Monthly } \\
\text { income } \\
(\text { EUR) }\end{array}$} & $<800$ & $19.0 \%$ & $22.8 \%$ \\
\hline & $801-2000$ & $39.7 \%$ & $35.8 \%$ \\
\hline & $2001-3000$ & $25.1 \%$ & $26.1 \%$ \\
\hline & $3001-4000$ & $8.8 \%$ & $7.3 \%$ \\
\hline & $>4000$ & $7.4 \%$ & $8.0 \%$ \\
\hline \multirow{5}{*}{$\begin{array}{l}\text { Educational } \\
\text { level }\end{array}$} & Intermediate mat. & $24.6 \%$ & $26.1 \%$ \\
\hline & High school & $26.0 \%$ & $21.1 \%$ \\
\hline & Vocational train. & $22.0 \%$ & $24.8 \%$ \\
\hline & Univ. degree & $26.9 \%$ & $27.5 \%$ \\
\hline & Other & $.5 \%$ & $.5 \%$ \\
\hline
\end{tabular}

\section{Results}

Before we share our results regarding our hypotheses, we elaborate on the data donation behavior variable, which we transformed to achieve a normal distribution. Furthermore, we checked that our manipulation regarding the mental state was successful. Afterward, we validated our measurement model. Finally, we ran a multi-group analysis (MGA) to test our hypotheses.

As our data for the actual data donation extent (i.e., how many pieces of information people donated) was not distributed normally (skewness $=-2.676$ and kurtosis=7.089), we calculated the fractional rank of the variable, resulting in uniformly distributed probabilities, and applied an inverse-normal transformation to form a variable of normally distributed z-scores [71]. The resulting skewness is .967 , and the kurtosis is .152 , which are within the range between \pm 2.0 for a normal distribution [30]. 
In order to check whether the manipulation of the participants' mental state (cold vs. hot) was successful, we first averaged the items based on the negative affect scale from PANAS-X [74]. Subsequently, we compared the negative affect between both groups using a t-test. We justify this approach with a sufficiently large sample size within each group [45]. We found that the negative affect is significantly higher in the COVID-19 group (mean=4.257; $\mathrm{sd}=1.474$; median=4.500) compared to the Ebola group (mean=3.314; sd=1.386; median=3.250) with $\mathrm{t}(445)=6.950, P<.001$. Thus, successful manipulation of the mental state can be assumed.

Finally, we compared the data donation extent between the COVID-19 and the Ebola group based on a t-test of the normalized variable. The mean value of donation behavior for the Ebola group is significantly higher than the COVID-19 sample with $\mathrm{t}(445)=-2.926$, $P=.004$. In particular, participants in the Ebola group donated on average 12.9 pieces of information, while participants in the COVID-19 group donated 12.0.

Before testing our hypotheses $\mathrm{H} 1$ and $\mathrm{H} 2$, we validated our measurement model for reliability, convergent validity, and discriminant validity. The Kaiser-Meyer-Olkin statistics (.890) confirmed that the data set is adequate for exploratory factor analysis. Concerning the item reliability, we checked the items' loadings with their respective constructs, which ranged between $.870-.953$ for privacy risks and .662 -.848 for trust and hence exceeded the threshold of .6 [56]. We assessed convergent validity and discriminant validity by checking that Cronbach's alpha is greater than .7 and composite reliability is at least .7 [7]. Moreover, the average variance extracted (AVE) should be higher than the threshold of .5 [7]. The following Table 3 shows that our measurement fulfills the requirements.

Table 3. Validity Measurements

\begin{tabular}{|l|l|l|l|}
\hline Construct & Cr. Alpha & Comp. Rel. & AVE \\
\hline Privacy Risk & .955 & .955 & .842 \\
\hline Trust & .876 & .899 & .642 \\
\hline
\end{tabular}

To analyze for discriminant validity in our model, we assessed the cross-loadings between the constructs. In this regard, the correlation between privacy risks and trust should not exceed .7 [28], which is not the case (.448). In sum, all scales for latent constructs possessed adequate reliability and validity.

As our main analysis, we evaluated the mental state's interaction and the model of privacy risks, trust, and data donation behavior. We created a model using SmartPLS representing privacy risks and trust as independent variables and data donation behavior as a dependent variable. The MGA is based on 5,000 bootstrapping iterations [18], differentiating between the COVID-19 group (hot state) and the Ebola group (cold state) (see Table 4). This approach is particularly suitable for analyzing heterogeneous data and two groups of equal sample size [60].

Table 4. Multi-Group-Analysis $(* P<.05)$

\begin{tabular}{|l|l|l|l|}
\hline Path & $\boldsymbol{\beta}$ (Ebola) & $\boldsymbol{\beta}($ COVID-19) & MGA (P) \\
\hline $\begin{array}{l}\text { Privacy Risk } \\
\rightarrow \text { Behavior }\end{array}$ & $-.159^{*}$ & $-.176^{*}$ & .87 \\
\hline $\begin{array}{l}\text { Trust } \\
\rightarrow \text { Behavior }\end{array}$ & $.269^{*}$ & -.028 & .01 \\
\hline
\end{tabular}

All control variables are insignificantly related to data donation behavior. Moreover, neither did the CMB marker significantly correlate with our dependent variable $(\beta=-.063 ; P=.445)$ nor did any regression path become insignificant. We, therefore, consider $\mathrm{CMB}$ not to be an issue in our data. Conducting the MGA showed that the effect of privacy risks on donation behavior is similarly strong in both groups $(P<.03)$. Hence, we need to reject $\mathrm{H} 1$, as the mental states do not interact with the influence of privacy risks on data donation behavior. In contrast, the influence of trust on data donation behavior varied, depending on the participants' mental state. Specifically, trust significantly affects data donation behavior only for the Ebola group (cold state) $(P=.03)$, while the path-coefficient is close to zero and not significant for the COVID-19 group (hot state) $(P=.73)$. These results support $\mathrm{H} 2$ since the mental state mitigates the influence of trust on data donation behavior. We report these results along with the pathcoefficients of each group in Table 4.

\section{Discussion}

Our study aims to investigate individuals' data donation decisions in light of a current pandemic. An experimental survey study among 445 participants showed that trust is not significantly linked to individuals' data donation decisions for the COVID-19 group (hot state). Participants solely focus on perceived privacy risks. As a result, the intense negative emotions attached to the COVID-19 pandemic lead to a lower data donation extent than a less emotionally induced donation purpose (Ebola). This is concerning as gathering real-time health data has become a major surveillance factor in combatting COVID-19.

Our study implies several practical contributions that can help research institutes, politicians, and individuals to better handle the COVID-19 pandemic in specific and further potential crises in general. First of all, confirming the results of other studies [75], the majority of our study's respondents donated a high amount of health data for research purposes. At first 
sight, this result is surprising as health data is considered very private and highly sensitive [9]. However, this might be the case since the data was donated to a scientific institution to support research. Indeed, previous studies show that people's willingness-to-disclose data for a good cause is higher than for commercial purposes [65]. However, what is concerning is that the data donation extent was higher to combat Ebola, which is a disease already under control and not pervasive in Europe. In particular, our results show that this is the case since people in a hot mental state caused by the research purpose COVID-19 are less able to decide rationally and narrow their attention to privacy risks when being asked to donate their health data. Based on strong negative emotions, they act rather egoistically than rationally. Even though trust might be rationally granted in the information recipient (e.g., a research institute) within a cold state, the actual willingness-todonate data in a hot state is not affected by trust as a driver for disclosure, mitigating the data donation extent. This is critical as health data, and its analysis have become an effective instrument to combat the crisis, for example, by identifying virus hot spots [62].

Since pandemics will continue to be possible in a globalized world, it is crucial to translate knowledge gained from crises experience and academic research into practice and start long-term preparations. To face possible future pandemics more effectively, we recommend establishing a persistent data donation platform on which people can provide their health data continuously and repeatedly and with a broader, potentially less negatively affected research purpose (e.g., combating viruses in general). Like blood donation, a one-time registration with a subsequent continuing affiliation could be initiated [15]. This way, data donation becomes more passive, and research institutes no longer need to actively encourage people to donate their data to combat an amid crisis, leading to a more defensive attitude or even reluctance regarding data donation. Furthermore, establishing a comprehensive dataset combining data from the pandemic state and from crisis-free periods might enable better analysis and respective countermeasures (e.g., early prediction of pandemic development) to prevent or combat pandemics. It is known from previous research that such data donation platforms have to comply with data protection regulations and be operated by a trustworthy institution (e.g., an independent research institution) [34]. In this sense, trust-promoting seals of approval, similar to the seals used by online stores or service providers [33], could promote data donation platforms' dissemination in a non-pandemic state.
In addition to the long-term goals, our research can also help find short-term remedies to combat the COVID-19 crisis. Our results suggest that individuals within the hot state of the COVID-19 pandemic narrow their attention to potential privacy risks, leading to a reduced willingness-to-donate. A possible countermeasure against this could be to emphasize the privacy friendliness of the research institute to lower perceived privacy risks and eventually promote data donation. This could be done, for example, in the form of an official statement from the institute in which its strict data practices are disclosed. Besides, institutes could make potential data donors aware of their biased decision-making process within a hot state to broaden their narrowed attention to factors other than risks (specifically trust). In particular, institutes should openly explain to potential donors how intense and pressing emotions could influence them in a hot state. Individuals need to cool off to bridge the gap between their mental states and make stable decisions independently of its emotional momentum. Governments could actively support this process, which can help move the population from a hot state to a cold state in which rational decision-making is possible. Thus, marking COVID-19 not only with negative emotions but also with positive feelings such as social cohesion, governmental resistance, and gratitude is necessary [10]. This could alleviate powerful negative emotions and keep individuals out of the hot state. In this regard, negative afflicted statements like "The situation is serious. Take it seriously, too." from the German chancellor Angela Merkel might fuel the high intensity of the population's negative emotions. In contrast, her statement, "I firmly believe that we can do this." might lower anxiety. As a result, even though fear appeals are common in crises, shaping the public's perception for good could be a key factor in collecting health data-especially in times when people are actively seeking governmental guidance, for example, in the form of financial support or official information [10].

Apart from practical contributions, our study's results provide several implications for theory on individuals' information disclosure behavior in general and privacy-related judgments in particular. The first overarching implication lies in investigating the interplay between individuals' actual willingness-todonate and emotions attached to the donation purpose. Secondly, we contribute to the literature by extending the privacy calculus with a critical behavioral biasnamely, an empathy gap. In this vein, we respond to a call of Dinev et al. (2015) [22] to test further nuances of the privacy calculus with regard to actual behavior and contribute to literature which questions the rational assumption of the privacy calculus $[2,3,35]$. 
We show that users' privacy decisions are biased, but not regarding privacy risks as previously demonstrated in other studies [13, 31, 72]. Instead, individuals consider trust differently depending on their mental state present at the time of decision formation. When in a hot state characterized by negative feelings such as anxiety or frustration, individuals' emotions override rational trust analysis. People narrow their attention to losses and fully concentrate on their risks. They act rather egotistically and are, in turn, unable to account for trust in the data-collecting institute. Even though their long-term goal might be to combat the spread of the virus, they focus on their short-term goal of reducing privacy risks in this specific situation. This behavior is caused by an inability to empathize with their cold state, which would lead to deliberate information processing and thus rational decisions as traditionally assumed in privacy research. Therefore, we argue that the impact of trust is so far insufficiently studied and should be considered more comprehensively as an essential driver of selfdisclosure. We support the notion that trust can be a key leveraging factor of self-disclosure online [49], particularly with regard to data donation in a cold state. However, our results explain why previous studies concerning the link between trust and self-disclosure have shown heterogeneous results. For instance, Norberg et al. (2007) [55] find that trust is not linked to self-disclosure. However, most other studies building on the privacy calculus theory conclude that trust is a major driver of self-disclosure [8, 9, 21, 49]. Our study sheds light on these contradicting results, which could stem from different situational factors associated with various mental states $[36,55]$.

Even though we provide several implications for theory and practice, our results are not free from methodological limitations. First, we conducted an experimental survey study by manipulating the mental state caused by the purpose of data donation. Although we controlled for a successful manipulation, varying data collecting purposes could have led to different results. Our second limitation lies within the operationalization of data donation behavior. We decided to measure the magnitude of actual data donation based on a self-developed catalog of 14 questions inspired by available COVID-19 applications. This specific set of questions may have influenced participants' donation behavior. However, measuring actual disclosure is seldom in research and questions the transferability of study results to real-life behavior. We counteract this issue by testing individuals' actual data donation decisions instead of asking for the intended willingness-to-donate.

\section{Conclusion}

In these times, public health data has become a critical surveillance factor in combating the spread of COVID-19. Data analytics heavily rely on data to run algorithms and thus identify ideal countermeasures. However, research analysts depend on massive amounts of public health data for such endeavors to be successful. Therefore, understanding how people make decisions about data donation in a crisis is of utmost importance. In other words: It is not only the technology itself that will turn the tide in this pandemic, but rather people's prosocial behavior. Recently, we have seen enormous selfless behavior with high societal consequences: People went shopping for the elderly, supported local shops, and kept their distance from loved ones. It is now the task of politics, research institutes, and each individual to transfer this empathy to the area of data donation.

\section{References}

[1] Acquisti, A., "Privacy in electronic commerce and the economics of immediate gratification", Proceedings of the ACM Conference on Electronic Commerce, Association for Computing Machinery (2004), 21-29.

[2] Acquisti, A., and J. Grossklags, "Privacy and rationality in individual decision making", IEEE Security and Privacy 3(1), 2005, pp. 26-33.

[3] Acquisti, A., L.K. John, and G. Loewenstein, "What is privacy worth?”, Journal of Legal Studies 42(2), 2013, pp. 249-274.

[4] Ågerfalk, P.J., K. Conboy, and M.D. Myers, "Information systems in the age of pandemics: COVID-19 and beyond", European Journal of Information Systems 29(3), 2020, pp. 203-207.

[5] Alashoor, T., N. Al-Maidani, and I. Al-Jabri, "The privacy calculus under positive and negative mood states", Proceedings of $39^{\text {th }}$ ICIS, (2018).

[6] Anderson, C.L., and R. Agarwal, "The digitization of healthcare: Boundary risks, emotion, and consumer willingness to disclose personal health information", Information Systems Research 22(3), 2011, pp. 469-490. [7] Bagozzi, R.P., and Y. Yi, "Specification, evaluation, and interpretation of structural equation models", Journal of Academy of Marketing Science 40(1), 2012, pp. 8-34. [8] Bansal, G., F.M. Zahedi, and D. Gefen, "The impact of personal dispositions on information sensitivity, privacy concern and trust in disclosing health information online", Decision Support Systems 49(2), 2010, pp. 138-150. [9] Bansal, G., F.M. Zahedi, and D. Gefen, "Do context and personality matter? Trust and privacy concerns in disclosing private information online", Information and Management 53(1), 2016, pp. 1-21.

[10] Bavel, J.J.V., K. Baicker, P.S. Boggio, et al., "Using social and behavioural science to support COVID-19 pandemic response", Nature Human Behaviour 4(5), 2020, pp. $460-471$. 
[11] Bietz, M., K. Patrick, and C. Bloss, "Data donation as a model for citizen science health research", Citizen Science: Theory and Practice 4(1), 2019, pp. 1-11. [12] Van Boven, L., and G. Loewenstein, "Empathy gaps in emotional perspective taking", In Other minds: How humans bridge the divide between self and others. Guilford Press, New York, NY, USA, 2005.

[13] Brakemeier, H., A. Wagner, and P. Buxmann, "When risk perceptions are nothing but guesses: An evaluability perspective on privacy risks", Proceedings of the 38 th ICIS, (2017).

[14] Brooks, S.K., R.K. Webster, L.E. Smith, et al., "The psychological impact of quarantine and how to reduce it: Rapid review of the evidence", The Lancet 395(10227), 2020, pp. 912-920.

[15] Cohen, E.L., and C. Hoffner, "Gifts of giving: The role of empathy and perceived benefits to others and self in young adults' decisions to become organ donors", Journal of Health Psychology 18(1), 2013, pp. 128-138.

[16] Culnan, M.J., and P.K. Armstrong, "Information privacy concerns, procedural fairness, and impersonal trust: An empirical investigation", Organization Science 10(1), 1999, pp. 104-115.

[17] Davis, M.H., "A multidimensional approach to individual differences in empathy", Journal of Personality and Social Psychology 10(85), 1980.

[18] Davison, A.C., and D. V. Hinkley, "Bootstrap

Methods and Their Application", Technometrics 42(2), 1997, pp. 216.

[19] Deutsch, M., "Trust and suspicion", Journal of Conflict Resolution 2(4), 1958, pp. 265-279.

[20] Dickert, S., and P. Slovic, "Attentional mechanisms in the generation of sympathy", Judgment and Decision Making 4(4), 2009, pp. 297-306.

[21] Dinev, T., and P. Hart, "An extended privacy calculus model for e-commerce transactions", Information Systems Research 17(1), 2006, pp. 61-80.

[22] Dinev, T., A.R. McConnell, H.J. Smith, T. Dinev, B. Raton, and H.J. Smith, "Informing privacy research through information systems, psychology, and behavioral economics: Thinking outside the 'APCO' box", Information Systems Research 26(4), 2015, pp. 639-655. [23] Dinev, T., H. Xu, J.H. Smith, and P. Hart, "Information privacy and correlates: An empirical attempt to bridge and distinguish privacyrelated concepts", European Journal of Information Systems 22(3), 2013, pp. 295-316.

[24] Drew, D.A., L.H. Nguyen, C.J. Steves, et al., "Rapid implementation of mobile technology for real-time epidemiology of COVID-19", Science 368(6497), 2020, pp. $1362-1367$.

[25] Eurostat, "Internet access and use statistics", 2018. [26] Everard, A., and D.F. Galletta, "How presentation flaws affect perceived site quality, trust, and intention to purchase from an online store", Journal of Management Information Systems 22(3), 2005, pp. 56-95.

[27] Fagherazzi, G., C. Goetzinger, M.A. Rashid, G.A. Aguayo, and L. Huiart, "Digital health strategies to fight COVID-19 worldwide", Journal of Medical Internet Research 22(6), 2020.

[28] Fornell, C., and D.F. Larcker, "Evaluating structural equation models with unobservable variables and measurement error", Journal of Marketing Research 18(1), 1981, pp. 39-50.

[29] France, C.R., and J.L. France, "Fear of donationrelated stimuli is reported across different levels of donation experience", Transfusion 58(1), 2018, pp. 113120 .

[30] Gao, S.P.L.M.R.A.J., "Non-normality of data in structural equation models", Journal of the Transportation Research Board 2082(1), 2008, pp. 116-124.

[31] Gerlach, J.P., P. Buxmann, T. Dinev, and P. Lowry, “"They're all the same!' Stereotypical thinking and systematic errors in users' privacy-related judgments about online services", Journal of the Association for Information Systems 20(6), 2019, pp. 787-823.

[32] Hanoch, Y., "“Neither an angel nor an ant': Emotion as an aid to bounded rationality", Journal of Economic Psychology 23(1), 2002, pp. 1-25.

[33] Hui, K., H. Kong, H.H. Teo, and S.T. Lee, "The value of privacy assurance: An exploratory field experiment", MIS Quarterly 31(1), 2007, pp. 19-33.

[34] Kaspar, K., "Motivation for social distancing and app use as complementary measures to combat the COVID-19 pandemic: Quantitative survey study", Journal of Medical Internet Research 22, 2020.

[35] Kehr, F., T. Kowatsch, D. Wentzel, and E. Fleisch, "Blissfully ignorant: The effects of general privacy concerns, general institutional trust, and affect in the privacy calculus", Information Systems Journal 25(6), 2015, pp. 607-635.

[36] Kehr, F., D. Wentzel, and P. Mayer, "Rethinking the privacy calculus: On the role of dispositional factors and affect", Proceeding of the 34th ICIS, (2013), 3355-3364. [37] Komiak, S.X., and I. Benbasat, "Understanding customer trust in agent-mediated electronic commerce, web-mediated electronic commerce, and traditional commerce", Information Technology and Management 5(1-2), 2004, pp. 181-207.

[38] Kopfman, J.E., and S.W. Smith, "Understanding the audiences of a health communication campaign: A discriminant analysis of potential organ donors based on intent to donate", Journal of Applied Communication Research 24(1), 1996, pp. 33-49.

[39] Krasnova, H., S. Spiekermann, K. Koroleva, and T. Hildebrand, "Online social networks: Why we disclose", Journal of Information Technology 25(2), 2010, pp. 109 125.

[40] Laufer, R.S., and M. Wolfe, "Privacy as a concept and a social issue: A multidimensional developmental theory", Journal of Social Issues 33(3), 1977, pp. 22-42.

[41] Li, H., X. (Robert) Luo, J. Zhang, and H. Xu, "Resolving the privacy paradox: Toward a cognitive appraisal and emotion approach to online privacy behaviors", Information and Management 54(8), 2017, pp. 1012-1022.

[42] Loewenstein, G., "Out of control: Visceral influences on behavior", Organizational Behavior and Human Decision Processes 65(3), 1996, pp. 272-292.

[43] Loewenstein, G., "Hot-cold empathy gaps and medical decision making", Health Psychology 24(4), 2005, pp. 49 56. 
[44] Lowry, P.B., J. D’Arcy, B. Hammer, and G.D. Moody, "'Cargo Cult' science in traditional organization and information systems survey research: A case for using nontraditional methods of data collection, including Mechanical Turk and online panels", Journal of Strategic Information Systems, 2016.

[45] Lumley, T., P. Diehr, S. Emerson, and L. Chen, "The importance of the normality assumption in large poblic health data sets", Annual Review of Public Health 23, 2002, pp. 151-169.

[46] Malhotra, N.K., S.S. Kim, and J. Agarwal, "Internet Users' Information Privacy Concerns (IUIPC): The Construct , the Scale, and a Causal Model", Information System Research 15(4), 2004, pp. 336-355.

[47] McKnight, D.H., V. Choudhury, and C. Kacmar, "Developing and validating trust measures for e-commerce: An integrative typology", Information Systems Research 13(3), 2002, pp. 334-359.

[48] Metcalfe, J., and W. Mischel, "A hot/cool-system analysis of delay of gratification: Dynamics of willpower", Psychological Review 106(1), 1999, pp. 3-19.

[49] Metzger, M.J., "Privacy, trust, and disclosure: Exploring barriers to electronic commerce", Journal of Computer-Mediated Communication 9(4), 2004.

[50] Möllering, G., "Rational, institutional and active trust: Just do it!?", In Trust under pressure: Empirical investigations of trust and trust building in uncertain circumstances. 2005, 17-36.

[51] Morgan, S.E., T.R. Harrison, S.D. Long, W.A. Afifi, M.S. Stephenson, and T. Reichert, "Family discussions about organ donation: how the media influences opinions about donation decisions", Clinical Transplantation 19(5), 2005, pp. 674-682.

[52] Murphy, M.D., D. Pinheiro, R. Iyengar, G. Lim, R. Menezes, and M. Cadeiras, "A data-driven social network intervention for improving organ donation awareness among minorities: Analysis and optimization of a crosssectional study", Journal of Medical Internet Research 22(1), 2020, pp. 1-15.

[53] Naidoo, R., "A multi-level influence model of COVID-19 themed cybercrime", European Journal of Information Systems 29(3), 2020, pp. 306-321.

[54] Norberg, P.A., and D.R. Horne, "Privacy attitudes and privacy-related behavior", Psychology \& Marketing 24(10), 2007, pp. 829-847.

[55] Norberg, P.A., D.R. Horne, and D.A. Horne, "The privacy paradox: Personal information disclosure intentions versus behaviors", Journal of Consumer Affairs 41(1), 2007, pp. 100-126.

[56] Nunnally, J.C., Psychometric theory, McGraw-Hill, New York, 1967.

[57] O'Guinn, T.C., and R.J. Faber, "Compulsive buying: A phenomenological exploration", Journal of Consumer Research 16(2), 1989, pp. 147.

[58] Pachur, T., R. Hertwig, and F. Steinmann, "How do people judge risks: Availability heuristic, affect heuristic, or both?", Journal of Experimental Psychology: Applied 18(3), 2012, pp. 314-330.

[59] Podsakoff, P.M., S.B. MacKenzie, J.Y. Lee, and N.P. Podsakoff, "Common method biases in behavioral research: A critical review of the literature and recommended remedies", Journal of Applied Psychology 88(5), 2003, pp. 879-903.

[60] Qureshi, I., H. Hom, H. Kong, and D. Compeau, "Assessing between-group differences in information systems research: A comparison of covariance- and component-based SEM", MIS Quarterly 33(1), 2009, pp. 197-214.

[61] Raghupathi, W., and V. Raghupathi, "Big data analytics in healthcare: Promise and potential", Health Information Science and Systems 2(1), 2014.

[62] RKI, "Corona data donation blog", 2020.

[63] Rowe, F., O. Ngwenyama, and J.-L. Richet, "Contacttracing apps and alienation in the age of COVID-19", European Journal of Information Systems, 2020.

[64] Siminoff, L., M.B. Mercer, G. Graham, and C. Burant, "The reasons families donate organs for transplantation: Implications for policy and practice", Journal of Trauma 62(4), 2007, pp. 969-978.

[65] Skatova, A., and J. Goulding, "Psychology of personal data donation", PLOS ONE 14(11), 2019.

[66] Skatova, A., E. Ng, and J. Goulding, "Data donation: Sharing personal data for public good?", Digital Economy 2014: Application of Digital Innovation, (2014). [67] Slovic, P., M. Finucane, E. Peters, and D.G. MacGregor, "Rational actors or rational fools: Implications of the affect heuristic for behavioral economics", The Journal of Socio-Economics 31(4), 2002, pp. 329-342. [68] Slovic, P., M.L. Finucane, E. Peters, and D.G. MacGregor, "The affect heuristic", European Journal of Operational Research 177(3), 2007, pp. 1333-1352. [69] Smith, J.H., T. Dinev, and H. Xu, "Information privacy research: An interdisciplinary review", MIS Quarterly 35(4), 2011, pp. 989-1015.

[70] Smith, S.P., R.B. Johnston, and S. Howard, "Putting yourself in the picture: An evaluation of virtual model technology as an online shopping tool", Information Systems Research 22(3), 2011, pp. 640-659. [71] Templeton, G.F., "A two-step approach for transforming continuous variables to normal", Communications of the Association for Information Systems 28(1), 2011, pp. 41-58.

[72] Wagner, A., and N. Mesbah, "Too confident to care: Investigating overconfidence in privacy desision-making", Proceedings of the 42th ECIS, (2019).

[73] Wakefield, R., "The influence of user affect in online information disclosure", Journal of Strategic Information Systems 22(2), 2013, pp. 157-174.

[74] Watson, D., and L.A. Clark, "The PANAS-X: manual for the positive and negative affect schedule", 1999, 1-24.

[75] Weitzman, E.R., L. Kaci, and K.D. Mandl, "Sharing medical data for health research: The early personal health record experience", Journal of Medical Internet Research 12(2), 2010

[76] Wessels, N., A. Wagner, J.P. Sarswat, and P. Buxmann, "What is your selfie worth? A field study on individuals" valuation of personal data", Proceedings of the 14th WI, (2019), 1322-1336.

[77] Xu, H., H.H. Teo, B.C.Y. Tan, and R. Agarwal, "The role of push-pull technology in privacy calculus", Journal of Management Information Systems 26(3), 2009, pp. 135174 